Bull. Mater. Sci., Vol. 21, No. 3, June 1998, pp. 203-206. (C) Indian Academy of Sciences.

\title{
Phase diagram and thermochemical properties of organic eutectic in a monotectic system
}

\author{
U S RAI* and R N RAI \\ Department of Chemistry, Banaras Hindu University, Varanasi 221005 , India
}

MS received 16 May 1997; revised 10 March 1998

\begin{abstract}
The phase diagram of a binary organic system involving diphenyl and succinonitrile shows the formation of a eutectic (0.968 mole fraction of succinonitrile) and a monotectic (0.074 mole fraction of succinonitrile) with a large miscibility gap in the system, the upper consolute temperature being $53.5^{\circ} \mathrm{C}$ above the monotectic horizontal. From the enthalpy of fusion of the pure components, the eutectic and the monotectic, determined by the DSC method, the enthalpy of mixing, Jackson's roughness parameter, interfacial energy, size of the critical nucleus and excess thermodynamic functions were calculated.
\end{abstract}

Keywords. Phase diagram; monotectic system; thermochemistry; organic eutectic; organic monotectic.

\section{Introduction}

The fundamental understanding of solidification (Elliott 1983; Herlach et al 1993; Glazer 1995; Rzyman et al 1996) and properties of polyphase alloys has become a subject of potential investigation during the past two decades. Due to low transformation temperature, ease in purification, transparency, wider choice of materials, minimized convection effects and simplicity of experimentation, organic systems (Glicksman et al 1983; Ecker et al 1989; Grugel and Poorman 1989; Podolinsky et al 1989; Sangster 1994; Trivedi and Kurz 1994; Favite 1996; Yasuda et al 1996) are better than metallic systems for scientific investigations. Succinonitrile is a low enthalpy of fusion material and solidifies like a metal. In contrast, diphenyl is a high enthalpy of fusion material and solidifies like a nonmetal. As such a system involving succinonitrile ( $\mathrm{SCN}$ ) and diphenyl (DP) is an organic analogue of metal-nonmetal system giving eutectic and monotectic alloys. Preliminary investigations on this system have already been published earlier (Rai and Rai 1996). However, in the absence of thermal data many aspects of this system could not be properly analysed. In view of this, phase diagram and thermochemistry of this system are reported here.

\section{Experimental}

\subsection{Materials and purification}

Diphenyl obtained from Sojuz Chem., Moscow was purified by fractional crystallization with ethanol. Succino-

*Author for correspondence nitrile (Aldrich, Germany) was purified by repeated distillation under vacuum. The purity of each compound was checked by determining melting point.

\subsection{Phase diagram}

Phase diagram of SCN-DP system was determined by the thaw-melt method (Rai and Mandal 1990). In this method mixtures of two components covering the entire range of composition are prepared in long-necked test tubes. The thawing and melting temperatures of each mixture were determined using a Toshniwal melting point apparatus attached with a thermometer which could read correctly up to $\pm 0.5^{\circ} \mathrm{C}$. A graph between melting temperature and composition gives the phase diagram.

\subsection{Enthalpy of fusion}

The values of enthalpy of fusion of each of the pure components, the eutectic and the monotectic, were determined by the DSC method using Mettler DSC-4000 system. Indium metal was used to calibrate the system. Amount of sample and rate of heating were about $5 \mathrm{mg}$ and $10^{\circ} \mathrm{C} / \mathrm{min}$, respectively, for each estimation. The maximum percentage error in the experimental value is 2 .

\section{Results and discussion}

\subsection{Phase diagram}

The phase diagram of SCN-DP system is given in figure 1. The melting temperature of diphenyl decreases with 
increase in concentration of succinonitrile. When concentration of succinonitrile is 0.074 mole fraction, liquid immiscibility appears (Rai and Rai 1996). At this composition which corresponds to $66.5^{\circ} \mathrm{C}$ melting temperature, two liquid phases, $L_{1}$ and $L_{2}$ and a solid phase are in equilibrium. This is the monotectic temperature and the composition corresponding to this temperature is the monotectic composition. When a liquid of monotectic composition is cooled below the monotectic temperature $\left(T_{\mathrm{m}}\right)$, the monotectic reaction occurs and a liquid, $L_{1}$ which is rich in DP decomposes into a solid phase $S$ (rich in DP) and another liquid $L_{2}$ (rich in SCN) according to the reaction,

$$
\left.L_{1}(\text { rich in } \mathrm{DP}) \rightleftharpoons \mathrm{DP}(S)+L_{2} \text { (rich in } \mathrm{SCN}\right) .
$$

When composition of succinonitrile is increased beyond 0.074 mole fraction, in a wide range area indicated by $L_{1}+L_{2}$, the two immiscible liquids are observed. At 0.968 mole fraction of succinonitrile there is a eutectic point and at this temperature two solid phases and a

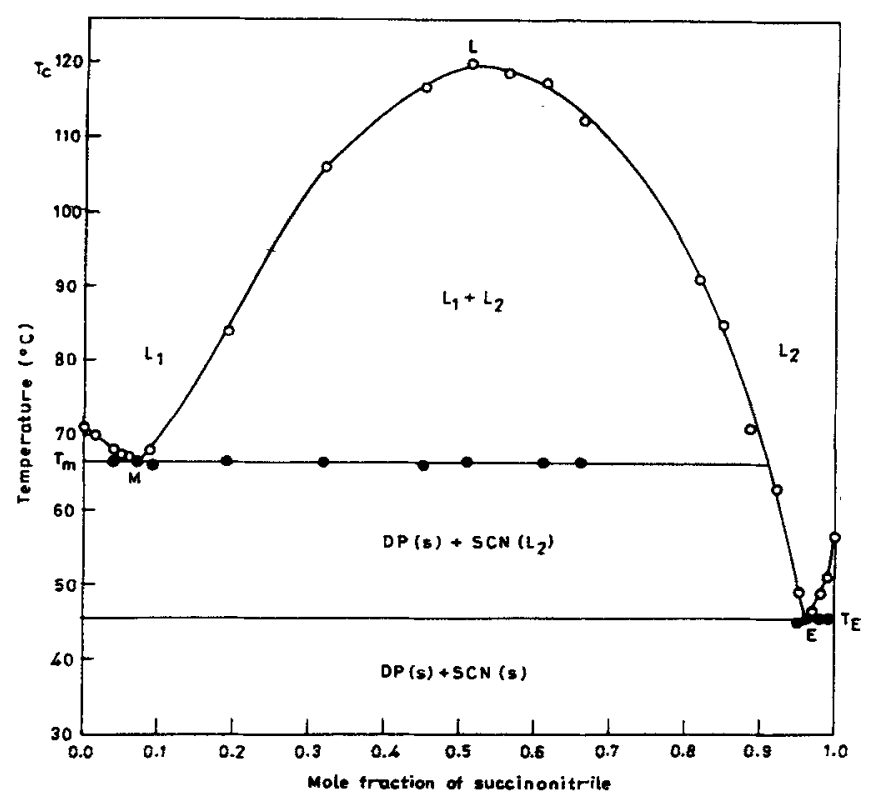

Figure 1. Phase diagram of diphenyl-succinonitrile system (o Melting temperature; - thaw temperature). liquid phase are in equilibrium. When a liquid of eutectic composition is allowed to cool below the eutectic temperature $\left(T_{\mathrm{E}}\right)$, the liquid decomposes to

$$
\begin{aligned}
L_{1}(\text { rich in } \mathrm{SCN}) \rightleftharpoons & S_{1}(\text { rich in } \mathrm{DP}) \\
& +S_{2}(\text { rich in } \mathrm{SCN}) .
\end{aligned}
$$

\subsection{Thermochemistry}

It is well known that phase transformation involves two steps: (i) nucleation, and (ii) growth. While the nucleation mechanism depends on the solid-liquid interfacial energy, the growth mechanism depends on the manner in which particles from the liquid phase are added at the solidliquid interface. The interface structure further depends on the entropy of fusion of the material under investigation and also upon the thermal environment in which the crystal is growing. Both the parameters, viz. interfacial energy and entropy of fusion can be calculated from the enthalpy of fusion data. Apart from this, radius of the critical nucleus, enthalpy of mixing, Jacksons roughness parameter and excess thermodynamic functions can also be calculated to throw light on the mechanism of solidification and formation of different microstructures of the eutectic and the monotectic.

3.2a Enthalpy of mixing: The values of enthalpy of fusion of the pure components, the eutectic and the monotectic, determined by the DSC method are given in table 1. For the purpose of comparison the enthalpy of fusion of the eutectic, calculated by the mixture law (Rai and George 1996), is also given in the same table. If the eutectic is a simple mechanical mixture involving no interaction, the value of enthalpy of fusion determined experimentally and calculated by the mixture law should be the same. The enthalpy of mixing $\left(\Delta_{\mathrm{m}} H\right)$ (Rai and Shekhar 1994), which is the difference between the experimental value of the enthalpy of fusion and the calculated value of the enthalpy of fusion, is $0.4 \mathrm{~kJ} \mathrm{~mol}^{-1}$ in the present system. Thermochemical studies (Singh et al 1985; Rai et al 1987) suggest that the structure of eutectic melt depends on the sign and the magnitude of the enthalpy of mixing. Three types of structures are suggested, quasi-eutectic for $\Delta_{\mathrm{m}} H>0$, clustering of mole-

Table 1. Heat of fusion, entropy of fusion and roughness parameter.

\begin{tabular}{lccc}
\hline Material & $\begin{array}{c}\text { Heat of } \\
\text { fusion } \\
\left(\mathrm{kJ} \cdot \mathrm{mol}^{-1}\right)\end{array}$ & $\begin{array}{c}\text { Entropy of } \\
\text { fusion } \\
\left(\mathrm{J} \cdot \mathrm{mol}^{-1}\right)\end{array}$ & $\begin{array}{c}\text { Roughness } \\
\text { parameter } \\
(\alpha)\end{array}$ \\
\hline Diphenyl (DP) & 18.5 & 53.8 & 6.5 \\
Succinonitrile (SCN) & 3.7 & $11 \cdot 2$ & 1.4 \\
DP-SCN eutectic (experimental) & 4.6 & 14.4 & 1.8 \\
DP-SCN eutectic (calculated) & $4 \cdot 2$ & $13 \cdot 2$ & 6.7 \\
DP-SCN monotectic (experimental) & 18.7 & $55 \cdot 1$ & 6.7 \\
\hline
\end{tabular}


Table 2. Interfacial energy of diphenyl, succinonitrile and their eutectic and monotectic.

\begin{tabular}{lc}
\hline Parameter & Value $\left(\mathrm{erg} \mathrm{cm}^{-2}\right)$ \\
\hline $\mathrm{SL}_{1}$ (DP) & $30 \cdot 3$ \\
$\mathrm{SL}_{2}$ (SCN) & 9.3 \\
$\mathrm{E}$ (DP-SCN) & 10.0 \\
$\mathrm{~L}_{1} \mathrm{~L}_{2}$ (DP-SCN) & 6.0 \\
\hline
\end{tabular}

cules for $\Delta_{\mathrm{m}} H<0$ and molecular solution for $\Delta_{\mathrm{m}} H=0$. The value of $\Delta_{\mathrm{m}} H$ being $+0.4 \mathrm{~kJ} \mathrm{~mol}^{-1}$, suggests quasi eutectic structure (Rai et al 1987) in the eutectic melt.

3.2b Entropy of fusion and excess thermodynamic functions: Theoretical studies on the entropy of fusion and calculation of some excess thermodynamic functions of eutectic predict the structure, stability and ordering in the eutectic melt. The values of entropy of fusion $\left(\Delta_{\mathrm{f}} S\right)$ of the pure components and eutectic were calculated using the equation,

$$
\Delta_{\mathrm{f}} S=\frac{\Delta_{\mathrm{f}} H}{T},
$$

where $\Delta_{\mathrm{f}} H$ is the enthalpy of fusion, $T$ the fusion temperature. The value of entropy of fusion being positive suggests that there is an increase in randomness during melting. Entropy factor is more effective in the eutectic in comparison to that of succinonitrile. On the other hand, it is less effective in comparison to that of diphenyl.

The deviation from ideal behaviour and quantitative measure of molecular interactions can be expressed in terms of excess thermodynamic functions. They are given by the difference between the thermodynamic function of mixing for a real system and the corresponding value for an ideal system at the same temperature and pressure.

$$
Y^{\mathrm{E}}=\Delta_{\text {mix }} Y(\text { real })-\Delta_{\text {mix }} Y(\text { ideal }),
$$

where $Y$ is any of the thermodynamic functions. These functions can be calculated using the method reported earlier (Rai et al 1983). The value of $Y^{\mathrm{E}}$ being positive $\left(210.7 \mathrm{~J} \mathrm{~mol}^{-1}\right.$ ) suggests (Wisniak and Tamir 1978) that associative interaction between like molecules is stronger than that between unlike molecules.

3.2c Interfacial energy and size of critical nucleus: When a liquid is cooled below its equilibrium melting temperature, the liquid phase does not solidify spontaneously because, under equilibrium condition, it contains clusters of molecules. So long as the clusters are all below the critical size (Christian 1965), they cannot grow to form crystals and so no solid is formed. The interfacial energy $(\sigma)$ is related (Singh 1978) to the critical size $\left(r^{*}\right)$ of the nucleus by the following equation:
Table 3. Radius of critical nucleus at different degrees of undercoolings.

\begin{tabular}{llll}
\hline & \multicolumn{3}{c}{ Critical radius $\left(\times 10^{8} \mathrm{~cm}\right)$} \\
\cline { 2 - 4 } $\begin{array}{l}\text { Undercooling } \\
\left(\Delta T^{\circ} \mathrm{C}\right)\end{array}$ & DP & SCN & Eutectic \\
\hline 2.5 & & 6.65 & \\
3.0 & & 5.55 & 4.62 \\
3.5 & & 4.75 & 3.96 \\
4.0 & & 4.16 & \\
4.5 & & 3.70 & 3.08 \\
5.0 & 2.25 & & 2.77 \\
5.5 & 2.05 & & \\
6.0 & 1.88 & & \\
7.0 & 1.61 & & \\
9.0 & 1.25 & & \\
\hline
\end{tabular}

$$
r^{*}=\frac{2 \cdot \sigma T_{\mathrm{m}}}{\Delta_{\mathrm{f}} H \cdot \Delta T},
$$

where $T_{\mathrm{m}}, \Delta_{\mathrm{f}} H$ and $\Delta T$ are melting temperature, heat of fusion and degree of undercooling, respectively. The interfacial energy is given by the relation

$$
\sigma=\frac{C \cdot \Delta_{f} H}{(N)^{1 / 3}\left(V_{\mathrm{m}}\right)^{2 / 3}},
$$

where $N$ is the Avogadro number, $V_{\mathrm{m}}$ the molar volume and parameter $C$ lies between 0.30 and 0.33 . The values of interfacial energy calculated using (6) are given in table 2. The critical size of the nucleus for pure components and the eutectics is given in table 3 . It is evident from the reported data that for a sample, the size of the critical nucleus decreases with an increase in the undercooling. This is due to an increase in the amplitude of vibration resulting from an increase in the undercooling.

\section{Acknowledgement}

Authors thank CSIR, New Delhi for providing financial assistance.

\section{References}

Christian J W 1965 The theory of phase transformations in metals and alloys (Oxford: Pergamon Press)

Ecker A, Frazier D O and Alexander J I D 1989 Metall. Trans. A20 2517

Elliott R 1977 Int. Met. Rev. 22161

Elliott R 1983 Eutectic solidification processing (London: Butterworths)

Favite G 1996 J. Cryst. Growth 16629

Glazer J 1995 Int. Mater. Rev. 4065

Glicksman M E, Singh N B and Chopra M 1983 Manufacturing in Space 11207

Grugel R N and Poorman R 1989 Mater. Sci. Forum 5089

Herlach D M, Cochrane R F, Egry I, Fecht H J and Greer A L 1993 Int. Mater. Rev. 38273 
Rai U S and Mandal K D 1990 Mol. Cryst. Liq. Cryst. 182387

Rai U S and Shekhar H 1994 Cryst. Res. Technol. 29551

Rai U S and George S 1996 J. Thermal Anal. 461809

Rai U S and Rai R N 1996 Thermochim. Acta 277209

Rai U S, Singh O P, Singh N P and Singh N B 1983 Thermochim. Acta 71373

Rai U S, Singh O P and Singh N B 1987 Can. J. Chem. 65 2639

Rzyman K, Moser Z, Watson R E and Weinert M $1996 \mathrm{~J}$. Phase Equilibria 17173
Sangster J 1994 J. Phys. Chem. Ref. Data 23295

Singh N B 1978 Acta Ciencia Indica 44

Singh N, Singh N B, Rai U S and Singh O P 1985 Thermochim. Acta 95291

Trivedi R and Kurz W 1994 Int. Mater. Rev. 3949

Wisniak $J$ and Tamir A 1978 Mixing and excess thermodynamic properties (A literature source book), in Phys. Sci. Data (New York: Elsevier)

Yasuda H, Ohnaka I, Matsunaga Y and Shiohara Y $1996 \mathrm{~J}$. Cryst. Growth 156128 